\title{
Transmissão Financeira entre o Mercado Acionário e o Mercado de Títulos de Dívida
}

\author{
*Francisco Eduardo de Luna e Almeida Santos
}

\begin{abstract}
Resumo
O objetivo do artigo é quantificar a relação endógena entre os retornos do mercado acionário e do mercado de títulos de dívida. Para recuperar os coeficientes estruturais dessa relação, empregou-se a metodologia de identificação pela heterocedasticidade. Verificou-se que ambos os coeficientes são negativos, o que está condizente com a noção de que, dado um custo de oportunidade de capital, os retornos devem se ajustar em direções opostas de forma a equilibrar o fluxo de capitais. Porém, somente o impacto dos retornos dos títulos de dívida nos retornos acionários foi diferente de zero no sentido estatístico. A intensidade dessa relação, portanto, também depende do tamanho relativo entre os mercados.
\end{abstract}

Palavras-chave: VAR estrutural, identificação, heterocedasticidade, mercado acionário, mercado de títulos.

Códigos JEL: E4, E5.

\section{Abstract}

The aim of this paper is to measure the endogenous relationship between stock and bond markets. To recover the structural form of this relationship, the author applied the method of identification through heteroskedasticity. Both coefficients were found to be negative which is consistent with the notion that, given an opportunity cost of capital, the returns move in opposite directions in order to promote the equilibrium of the capital flow. However, only the coefficient that measures the impact of bond market over stock markets was significantly different from zero. Thus, the intensity of this relationship also depends on the relative size of the markets under study.

Keywords: structural VaR, identification, heteroscedasticy, stock market, securities market.

\section{Introdução}

A relação entre o movimento de ativos financeiros, apesar de inequívoca, carece de consenso quanto à magnitude e períodos em que se manifesta. Como os recursos financeiros se movem livremente entre esses ativos, impulsionados tanto por fatores econômicos da economia real como por expectativas individuais, a

Submetido em Junho de 2007. Aceito em Abril de 2008. O artigo foi avaliado segundo o processo de duplo anonimato além se de ser avaliado pelos editores. Editor principal: Ricardo P. C. Leal. As opiniões expressas neste artigo são de inteira responsabilidade do autor e não expressam a visão do Banco Central do Brasil e nem de sua diretoria. O autor agradece os colegas do Instituto de Pesquisa Econômica Aplicada e do Banco Central do Brasil pelas valiosas contribuições e comentários.

*Analista do Departamento de Mercado Aberto no Banco Central do Brasil. Avenida Presidente Vargas, 730, $6^{\circ}$ andar, Centro, Rio de Janeiro, Brasil. CEP: 20071-900. Tel: (21) 2189-5244 / (21) 9501-5504. E-mail: francisco.luna@superig.com.br 
modelagem dessas decisões de investimento está se tornando cada vez mais complexa. Uma análise geral desse fluxo, considerando somente os principais agregados econômicos, permite, ao menos, identificar algumas relações básicas.

Mais recentemente, no Brasil, o mercado acionário tornou-se opção bastante viável de financiamento, oferecendo boa liquidez e possibilidades de compartilhamento de riscos. Em 2007, a captação totalizou R $\$ 33,1$ bilhões por meio de 59 distribuições públicas de ações e apresenta tendência crescente desde 2003, ainda que os montantes anuais sejam diretamente influenciados pela volatilidade do mercado. Esse desempenho se reflete no valor de mercado das empresas com ações negociadas na Bovespa, que alcançou R \$ 2,48 trilhões em dezembro de 2007.

O mercado de títulos, por sua vez, segue bastante atrelado aos movimentos de política monetária, em especial a partir da implantação do regime de câmbio flutuante e das metas inflacionárias, em 1999. A captação via debêntures, por exemplo, foi de aproximadamente $\mathrm{R} \$ 46,5$ bilhões em 2007, enquanto que o mesmo montante foi de R $\$ 69,5$ bilhões em 2006. A manutenção da estabilidade monetária e a ação mais previsível do Banco Central favorecerão o crescimento desse mercado e permitirão o estabelecimento de uma estrutura a termo de taxa juros mais robusta.

Uma linha de pesquisadores se concentrou nos efeitos das taxas de juros sobre o mercado acionário. O cálculo do preço teórico de uma ação a partir da série de dividendos requer a utilização de taxas de desconto dos fluxos de caixa da firma. As taxas de juros de longo prazo refletem a expectativa dos agentes econômicos quanto à atuação da política monetária no futuro. Expectativas negativas quanto à inflação provocam aumentos na taxa de juros de longo prazo e no fator de desconto o que, em tese, reduziria o valor presente do fluxo de dividendos. A relação inversa, ou seja, do mercado acionário no mercado de títulos, é mais indireta, porém não menos importante. Segundo Bernanke e Gertler (2001), os retornos acionários só deveriam influenciar a política monetária na medida em que afetarem a expectativa futura de inflação.

Nesse contexto, estudar-se-á a relação entre o mercado acionário e o de títulos de dívida. Faz parte do cálculo de custo de capital de uma empresa a ponderação entre o custo de financiamento por meio de lançamento de ações ou dívida. $\mathrm{O}$ maior entendimento da transmissão de choques entre esses mercados, portanto, forneceria maiores subsídios para a tomada de decisão de investimento e financiamento das empresas, motivação principal do presente artigo. Dada a natureza endógena e contemporânea da integração entre esses mercados, é objetivo desse artigo identificar os coeficientes estruturais dessa relação. Para tal fim, empregou-se a metodologia de identificação pela heterocedasticidade (IH), descrita em detalhes no anexo.

\section{Metodologia e Modelo}

Dada a relação endógena do movimento no preço de ativos financeiros, a abordagem VAR é normalmente utilizada. Um dos desafios na aplicação da metodologia VAR é superar o problema da identificação da forma estrutural do modelo. Em 
grande parte dos trabalhos empíricos, o modelo é estimado em sua forma reduzida e, sobre a estrutura de inovações, é empregada a Decomposição de Choleski permitindo, assim, a obtenção dos resultados analíticos, como a função impulsoresposta e a decomposição de variância. Porém, nem sempre é possível interpretar economicamente as restrições impostas por essa triangularização da matriz de inovações de forma que métodos alternativos surgiram para melhorar o processo de identificação, como a metodologia $\mathrm{IH}$, que utiliza mudanças na estrutura de covariância das séries para identificar os choques estruturais.

Essa alternativa de identificação foi utilizada em alguns trabalhos de finanças internacionais e política monetária. Rigobon (2000) empregou essa metodologia no estudo do contágio entre títulos soberanos de países emergentes. Da mesma forma, Rigobon e Sack (2001) analisaram o impacto do preço das ações na política monetária nos Estados Unidos e identificaram que aumentos no preço dos ativos provocam aumentos na taxa de curto prazo. Mais recentemente, Ehrmann et alii (2005) estudaram os mecanismos de transmissão financeira entre classes de ativos nos Estados Unidos e Europa por meio da metodologia IH.

O método de IH impõe duas premissas para identificação do modelo: em primeiro lugar, os choques estruturais são não-correlacionados e os parâmetros estruturais são estáveis no período amostral. A introdução de um fator comum nãoobservável permite que separemos o resíduo do VAR em uma parte correlacionada e outra não correlacionada. Na prática, estamos admitindo que o controle das variáveis exógenas não é perfeito e o choque estrutural apresentaria alguma correlação na ausência dessa separação.

As variáveis endógenas do modelo são representativas do mercado acionário e do mercado de títulos de dívida. No primeiro caso, a escolha natural é o retorno do índice Bovespa (BOV), que agrega a maior parte das empresas que listam ações em bolsa de valores. Para representar o movimento do mercado de títulos de dívida, é necessário escolher a taxa de juros de longo prazo. Na estrutura a termo brasileira, a taxa de 12 meses (Ettj12m) possui boa liquidez e pode ser utilizada para tal fim. Essa taxa é um importante indicador das expectativas de inflação, pois indicam como será a atuação futura da política monetária.

Além das variáveis domésticas, a transmissão pode ocorrer por meio de eventos que afetam a liquidez externa ou aumentam a aversão a risco em emergentes. Um aumento na taxa de juros norte-americanos, por exemplo, provoca uma realocação de carteiras, e alguns investidores migram suas aplicações para ativos de menor risco, como os Treasury Bonds, com efeitos diretos no preço das ações no Brasil. Ao modelo, foram acrescentados dois fatores comuns exógenos: o risco-país (EMBI) e os retornos do SP\&500 (SP500), importante índice da bolsa norte-americana.

\section{Resultados}

No presente trabalho, a frequiência de dados será diária e o período de análise se estendeu de 01/09/1999 até 31/12/2006, totalizando 1860 observações. O pri- 
meiro passo para a aplicação do método de IH consiste em estimar o VAR na forma reduzida. Testes preliminares de estacionaridade (Dickey-Fuller Aumentado e Philipps-Perron) indicaram que a taxa de juros de longo prazo deve ser utilizada em primeiras diferenças, e os retornos dos índices Bovespa, SP\&500 e Embi já são estacionários. Ademais, os testes de AIC, HQ e FPE recomendaram a introdução de duas defasagens nas variáveis dependentes. Feitas as considerações acima, os resultados do VAR são apresentados na tabela a seguir.

Tabela 1

Resultados da aplicação do VAR

\begin{tabular}{lcc}
\hline Coeficientes & Equação para r(Bov) & Equação para d(Ettj12m) \\
\hline Intercepto & $0,001^{* *}$ & $<0,001^{N S}$ \\
$r(B o v)$ em $t-1$ & $-0,053^{* * *}$ & $-0,004^{N S}$ \\
$r(B o v)$ em $t-2$ & $-0,032^{N S}$ & $-0,001^{N S}$ \\
$d(E t t j 12 m)$ em $t-1$ & $0,113^{*}$ & $-0,014^{N S}$ \\
$d(E t t j 12 m)$ em $t-2$ & $-0,152^{N S}$ & $-0,094^{* * *}$ \\
$r(E m b i)$ & $-0,336^{* * *}$ & $0,073^{* * *}$ \\
$r(S P 500)$ & $0,618^{* * *}$ & $-0,014^{* *}$ \\
$R^{2}$ ajustado & 0,43 & 0,27 \\
\hline Nota: ***: Significativo a 1\%; **: Significativo a 5\%; *: Significativo a 10\%; &
\end{tabular}

Os coeficientes da equação para o retorno do Bovespa são significativos, com exceção das variáveis dependentes defasadas $t-2$. Soma-se a isso o fato de os fatores comuns apresentarem o sinal esperado. Analisando a equação para a taxa de juros de longo prazo, vemos que os coeficientes dos retornos acionários defasados não influenciam, em termos estatísticos, a formação dessa taxa. Mais adiante, verificamos a natureza da relação contemporânea entre as variáveis endógenas. Assim como na equação anterior, os coeficientes dos fatores comuns são significativos e apresentam os sinais teóricos esperados. Em ambos os casos, o bom nível de $R^{2}$ é um indicador adicional que corrobora a qualidade do ajuste da regressão.

Quanto aos resíduos, a análise das correlações não fornece indícios da presença de correlação serial e os testes de estacionaridade mostram que são estacionários. Essa análise é particularmente importante tendo em vista que os resíduos são o insumo para a próxima etapa da metodologia de IH. Como apresentam boas propriedades, é possível utilizá-los para a definição dos regimes de covariância e, posteriormente, no cálculo dos coeficientes estruturais.

Para a identificação dos coeficientes estruturais, é necessário separar a amostra dos resíduos em, no mínimo, três regimes. Essa separação poderia ser feita por meio da definição de períodos em que sabidamente ocorreram mudanças de volatilidade das variáveis de interesse. Períodos de crise, como o "apagão" elétrico em 2001, as eleições em 2002 e a crise política de 2005 seriam os candidatos naturais. Porém, as variâncias e covariâncias nos diferentes regimes não se alteraram de modo satisfatório. Isso se deve, provavelmente, à dificuldade em definir o momento exato de corte para a divisão da amostra uma vez que estamos lidando com dados em freqüência diária. 
A alternativa, fundamentada no trabalho de Rigobon e Sack (2001), é utilizar a variância dos resíduos da própria amostra em questão. Dessa forma, é possível separá-la em quatro regimes: 1) ambos os resíduos têm baixa volatilidade; 2) somente o resíduo do retorno acionário tem alta volatilidade; 3) Somente o resíduo da taxa de longo prazo tem alta volatilidade; 4) Ambos têm alta volatilidade. A volatilidade é considerada alta se a variância calculada para uma janela de 22 dias úteis (aproximadamente um mês) ultrapassar, por mais de um desvio-padrão, a média das variâncias da série.

A divisão da amostra em quatro partes permite a comparação dos coeficientes estruturais para combinações distintas de regimes. Além disso, por se tratar de procedimento essencialmente arbitrário, é fundamental testar configurações alternativas de janela a fim de avaliar sua robustez. Para esse fim, alteraremos a distância da média na definição anterior de alta volatilidade.

A tabela a seguir, mostra a matriz de covariância para os regimes em consonância com os critérios acima expostos.

Tabela 2

Dados de variância e covariância dos regimes selecionados

\begin{tabular}{lccc}
\hline Regime & $\sigma^{2}(r($ Bov $))$ & $\sigma^{2}(\mathrm{~d}(\mathrm{Ettj} 12 \mathrm{~m}))$ & $\operatorname{Cov}(d(\operatorname{Ettj} 12 \mathrm{~m}), r($ Bov $))$ \\
& $* 10^{4}$ & $* 10^{5}$ & $* 10^{5}$ \\
\hline 1 & 1,63 & 0,54 & $-0,75$ \\
2 & 3,15 & 0,49 & $-0,83$ \\
3 & 1,94 & 3,44 & $-2,64$ \\
4 & 3,67 & 6,43 & $-4,95$ \\
\hline
\end{tabular}

É importante notar que o regime 1, que representa períodos mais tranqüilos, agrega a maior parte das observações e os períodos de crise, em que ambos os resíduos apresentam alta volatilidade, são mais raros.

Tabela 3

Participação relativa de cada regime na quantidade total de observações

\begin{tabular}{lc}
\hline Regime & Participação \\
\hline 1 & $72,25 \%$ \\
2 & $10,77 \%$ \\
3 & $5,38 \%$ \\
4 & $4,07 \%$ \\
\hline
\end{tabular}

De maneira geral, a quantidade de observações em cada regime é bastante adequada para a implementação do passo seguinte: o cálculo da equação quadrática para obter os coeficientes estruturais. Utilizando as informações da matriz de covariância, foram geradas 1000 amostras de resíduos por meio de bootstrap. Com isso, obtém-se a média e o desvio-padrão dos coeficientes. 
O coeficiente estrutural $\alpha$ mede o efeito contemporâneo do mercado acionário no mercado de títulos de dívida. Esse efeito se faz presente na medida em que o fluxo de capitais entre ativos financeiros é intenso, motivado por alterações na percepção de risco e retorno entre os mesmos. Espera-se que, devido ao peso relativo entre os mercados em questão, o mercado acionário exerça influência menor no mercado de títulos do que o contrário. A tabela a seguir apresenta as medidas de $\alpha$ para diferentes combinações de regimes.

Tabela 4

Resultados para o coeficiente

\begin{tabular}{lccc}
\hline $\begin{array}{c}\text { Combinação de } \\
\text { regimes }\end{array}$ & Média & Desvio-padrão & $\begin{array}{c}\text { Percentual de } \\
\text { observações negativas }\end{array}$ \\
\hline$\alpha_{123}$ & $-0,007$ & 0,020 & 52,9 \\
$\alpha_{124}$ & $-0,007$ & 0,020 & 55,7 \\
$\alpha_{134}$ & $-0,033$ & 0,218 & 57,8 \\
$\alpha_{234}$ & $-0,017$ & 0,099 & 58,0 \\
\hline
\end{tabular}

A média das combinações é de -0,016, de modo que um choque não esperado de $1 \%$ no retorno acionário provoca uma queda de $0,016 \%$ na taxa de juros de longo prazo. Os valores médios de $\alpha$ apresentam boa estabilidade e, em todos os casos, é negativo. Porém, estatisticamente não é possível afirmar que o coeficiente seja diferente de zero dado o alto desvio-padrão das medidas e uma divisão aproximadamente equânime entre valores negativos e positivos.

Como uma das premissas de IH é a estabilidade dos coeficientes estruturais, realizou-se um teste de diferença de médias para averiguar sua aplicabilidade. Os testes mostram que, em somente uma das comparações, podemos afirmar que os coeficientes são iguais. Portanto, existe alguma não-linearidade que não foi captada na aplicação do método.

Tabela 5

Intervalos de confiança para a diferença de médias dos resultados do coeficiente

\begin{tabular}{ccc}
\hline & Inferior & Superior \\
\hline$\alpha_{123}-\alpha_{124}$ & $-0,001$ & 0,002 \\
$\alpha_{123}-\alpha_{134}$ & 0,013 & 0,041 \\
$\alpha_{123}-\alpha_{234}$ & 0,004 & 0,016 \\
$\alpha_{124}-\alpha_{134}$ & 0,013 & 0,040 \\
$\alpha_{124}-\alpha_{234}$ & 0,004 & 0,016 \\
$\alpha_{134}-\alpha_{234}$ & $-0,032$ & $-0,002$ \\
\hline
\end{tabular}

Para contornar esse problema, modificamos a configuração dos regimes adotando duas novas distâncias em relação à média na definição de alta volatilidade: 0,75 e 0,5 desvio-padrão. No primeiro caso, a média de $\alpha$ foi idêntica $(-0,017)$ e, no segundo caso, foi de $-0,008$. Percebe-se que o coeficiente é bastante robusto a alterações nas definições do procedimento. Feitas as devidas ressalvas, portanto, pode-se atestar que o método de IH é perfeitamente aplicável e que aumentos (reduções) no retorno do mercado acionário provocam reduções (aumentos) de baixa intensidade na taxa de juros de longo prazo. 
Ehrmann et alii (2005) analisaram o grau de transmissão financeira entre três classes de ativos (taxas de juros de curto e longo prazo; e ações) nos Estados Unidos e União Européia. Nos Estados Unidos, um aumento de $1 \%$ nos retornos acionários implica em redução de $0,01 \%$ nas taxas de longo prazo, representante do mercado de títulos. Na Europa, essa influência existe, em menor escala, e não é significativa. Além do componente doméstico, há fortes evidências de transbordamento de choques de preços entre as classes de ativos e entre os países. Choques em ativos dos Estados Unidos explicam, em média, 25\% dos choques nos ativos europeus enquanto que, no sentido inverso, a capacidade explicativa cai para $8 \%$.

Em teoria, a capitalização de mercado deveria refletir o preço justo de seus ativos. O indicador preço/lucro é comumente utilizado para referenciar o nível de valorização entre empresas ou países. Tradicionalmente, essa relação varia em torno de dez no Brasil e, mais recentemente, está próximo de quinze. Chile e México, por exemplo, têm uma relação maior, mais compatível com a dos países desenvolvidos. Ainda que consideremos as limitações dessa medida, havia, no período amostral, espaço para valorização das ações sem que isso representasse sobrevalorização ou um aquecimento dos demais preços da economia que, somada ao baixo percentual de capitalização, constituem possíveis justificativas para a baixa intensidade da influência do mercado acionário brasileiro no mercado de títulos. Como essa valorização é normalmente acompanhada por transferência de fluxos financeiros de renda fixa para variável, e supondo que o custo de oportunidade $^{1}$ do capital se mantenha constante no curto prazo, é natural que haja redução na taxa de juros de longo prazo de forma a equilibrar os mercados, ainda que a intensidade desse efeito seja baixa.

O coeficiente estrutural $\beta$ mede o efeito contemporâneo do mercado de títulos de dívida sobre o mercado acionário. É razoável supor que aumentos de taxa de juros diminuam a atratividade de investimentos em renda variável, pois, neste caso, o fluxo de capitais se direcionará para o mercado com a melhor relação entre risco e retorno. Os resultados mostrados na tabela 6 apontam para essa realidade.

Tabela 6

Resultados para o coeficiente

\begin{tabular}{lccc}
\hline $\begin{array}{c}\text { Combinação de } \\
\text { regimes }\end{array}$ & Média & Desvio-padrão & $\begin{array}{c}\text { Percentual de } \\
\text { observações negativas }\end{array}$ \\
\hline$\alpha_{123}$ & $-0,652$ & 0,261 & 99,1 \\
$\alpha_{124}$ & $-0,711$ & 0,303 & 99,1 \\
$\alpha_{134}$ & $-0,549$ & 0,533 & 90,3 \\
$\alpha_{234}$ & $-0,702$ & 0,308 & 98,1 \\
\hline
\end{tabular}

\footnotetext{
${ }^{1} \mathrm{O}$ teorema de Modigliani \& Miller trata a forma de financiamento como uma variável passiva, ou seja, havendo projetos cujo retorno seja compensador, a empresa não teria dificuldades na obtenção de recursos.
} 
A média do coeficiente $\beta$ entre toda as combinações é de - 0,654 de modo que um aumento linear não esperado de $0,5 \%$ na taxa de longo prazo provoca uma redução média de $0,327 \%$ no retorno acionário. Os valores apresentam boa estabilidade e, com exceção do coeficiente $\beta_{134}$, é possível afirmar que sejam diferentes de zero a um nível de significância de 5\%. Em todos os casos, mais de $90 \%$ das observações são negativas.

Realizou-se um teste de diferença de médias para atestar a estabilidade entre os coeficientes. Os testes mostram que, em somente uma das comparações, podemos afirmar que os coeficientes são iguais. Portanto, assim como nos resultados para o coeficiente $\alpha$, a aplicação do método deixa de captar alguma não-linearidade no sistema.

Tabela 7

Intervalos de confiança para a diferença de médias dos resultados do coeficiente

\begin{tabular}{ccc}
\hline & Inferior & Superior \\
\hline$\beta_{123}-\beta_{124}$ & 0,034 & 0,084 \\
$\beta_{123}-\beta_{134}$ & $-0,141$ & $-0,067$ \\
$\beta_{123}-\beta_{234}$ & 0,024 & 0,074 \\
$\beta_{124}-\beta_{134}$ & $-0,201$ & $-0,124$ \\
$\beta_{124}-\beta_{234}$ & $-0,036$ & 0,017 \\
$\beta_{134}-\beta_{234}$ & 0,115 & 0,191 \\
\hline
\end{tabular}

Novamente, modificamos a configuração dos regimes adotando duas novas distâncias em relação à média na definição de alta volatilidade: 0,75 e 0,5 desviopadrão. No primeiro caso, a média de $\beta$ foi de $-0,577$ e, no segundo caso, foi de $-0,623$. Com isso, confirmamos que eventuais não-linearidades não afetam drasticamente o resultado dos coeficientes, condição necessária para a aplicação do método de IH. Portanto, pode-se afirmar, apoiado na robustez do método, que aumentos (reduções) na taxa de juros de longo prazo provocam reduções (aumentos) no retorno do mercado acionário.

Ehrmann et alii (2005) apontam que o mercado de ações norte-americano não reage significativamente a movimentos na taxa de longo prazo. Por outro lado, na Europa, aumentos de $0,5 \%$ na taxa de juros promovem reduções de $0,26 \%$ no mercado acionário. Quando se considera a taxa de curto prazo, esse efeito é negativo e significativo em ambos os casos.

\section{Conclusão}

A estimativa de um modelo VAR na forma reduzida pode ser bastante útil para fins de análise de correlações, além de fornecer previsões ótimas. Contudo, perdem-se informações acerca do movimento contemporâneo entre as variáveis uma vez que os resíduos dessa estimativa não têm qualquer interpretação econômica. 
O presente artigo empregou a metodologia IH para identificar a forma estrutural da relação entre os retornos dos mercados acionários e de títulos. Mudanças na estrutura de covariância dos próprios dados permitem essa identificação. A crescente integração e a livre movimentação de capitais entre esses mercados justificam a necessidade de se recuperar a forma estrutural dessa relação.

Verificou-se que o impacto contemporâneo do mercado acionário no mercado de títulos de dívida é negativo e de baixa intensidade. Um choque positivo de $1 \%$ no retorno do Ibovespa provoca, em média, uma queda de $0,016 \%$ na taxa de juros de longo prazo. Esses resultados são consistentes com os obtidos em outros estudos empíricos internacionais e com algumas características peculiares do mercado brasileiro apontadas no estudo.

A relação inversa se manteve negativa; porém, ao contrário do caso anterior, é diferente de zero no sentido estatístico. Um aumento linear não esperado de $0,5 \%$ na taxa de longo prazo provoca uma redução média de $0,327 \%$ no retorno acionário.

Ambos os coeficientes confirmam a noção de que os retornos devem se mover em direções opostas para equilibrar o fluxo de capitais. Porém considerando o tamanho relativo entre os mercados, esse efeito é mais intenso quando medimos o impacto da taxa de longo prazo sobre os retornos acionários.

Em ambos os casos, os resultados foram relativamente robustos a alterações na definição dos regimes de covariância; porém, o teste de diferença de médias aponta que há não-linearidades não captadas pelo modelo. Ademais, vale a ressalva de que a interpretação dos coeficientes parte do pressuposto de que os fatores comuns estão controlados pelo modelo, eliminando, desta forma, efeitos indiretos nos coeficientes estruturais. Considerando o exposto acima, esse artigo provê importantes informações para entender o movimento contemporâneo entre dois mercados de suma importância no contexto do sistema financeiro nacional.

\section{Referências}

Bernanke, B. S. \& Gertler, M. (2001). Should central banks respond to movements in asset prices? The American Economic Review, 91(2):253-257.

Ehrmann, M., Fratzscher, M., \& Rigobon, R. (2005). Stocks, bonds, money markets and exchange rates: Measuring international financial transmission. NBER Working Paper 11166, National Bureau of Economic Research, Inc.

Rigobon, R. (2000). Identification through heteroskedasticity: Measuring contagion between Argentinean and Mexican sovereign bonds. NBER Working Paper 7493, National Bureau of Economic Research, Inc.

Rigobon, R. \& Sack, B. (2001). Measuring the reaction of monetary policy to the stock market. NBER Working Paper 8350, Cambridge. 


\section{Anexo}

\section{Método Identificação pela Heterocedasticidade (IH)}

Um modelo VAR na forma estrutural pode ser representado da seguinte forma: $A . Y_{t}=\phi(L) . Y_{t}+\varphi \cdot X_{t}+\delta . Z_{t}+\mu_{t}$, onde $Y_{t}, X_{t}$ e $Z_{t}$ são compostas pelas variáveis endógenas, pelos fatores comuns observáveis e não-observáveis, respectivamente. O operador $\phi(L)$ introduz as defasagens nas variáveis endógenas e as matrizes $\varphi$ e $\delta$ representam os coeficientes dos fatores comuns observáveis. A matriz A se refere aos parâmetros estruturais e $\mu_{t}$, aos choques estruturais.

No caso de duas variáveis endógenas e um fator comum não-observável, os parâmetros assumem a seguinte representação:

$$
A=\left(\begin{array}{cc}
1 & \beta \\
\alpha & 1
\end{array}\right), \delta=\left(\begin{array}{l}
1 \\
\gamma
\end{array}\right) \mu_{t}=\left(\begin{array}{l}
\epsilon_{t} \\
\eta_{t}
\end{array}\right)
$$

A passagem para a forma reduzida implica em multiplicar ambos os lados da equação estrutural por $A^{-1}$, resultando na seguinte equação:

$$
Y_{t}=A^{-1} \phi(L) \cdot Y_{t}+A^{-1} \varphi \cdot X_{t}+A^{-1} \delta \cdot Z_{t}+A^{-1} \mu_{t}
$$

As duas últimas expressões do lado direito da equação anterior compõem os resíduos do VAR na forma reduzida. Assumindo que os choques estruturais são não-correlacionados, a matriz de covariância desses resíduos pode ser escrita como:

$$
\begin{gathered}
\Omega_{r e s}=\frac{1}{(1-\alpha \beta)^{2}} \\
\left(\begin{array}{cc}
(\beta+\gamma)^{2} \sigma_{z}^{2}+\beta^{2} \sigma_{\eta}^{2}+\sigma_{\epsilon}^{2} & (1+\alpha \gamma)(\beta+\gamma) \sigma_{z}^{2}+\beta \sigma_{\eta}^{2}+\alpha \sigma_{\epsilon}^{2} \\
(1+\alpha \gamma)(\beta+\gamma) \sigma_{z}^{2}+\beta \sigma_{\eta}^{2}+\alpha \sigma_{\epsilon}^{2} & (1+\alpha \gamma)^{2} \sigma_{z}^{2}+\sigma_{\eta}^{2}+\alpha^{2} \sigma_{\epsilon}^{2}
\end{array}\right)
\end{gathered}
$$

A matriz de covariância tem 6 incógnitas, a saber: $\alpha, \beta, \gamma, \sigma_{e p s i l o n}^{2}, \sigma_{\mu}^{2}$ e $\sigma_{z}^{2}$. De acordo com o método de IH, a divisão da amostra em regimes de covariância fornece as informações necessárias para identificar a solução do sistema. Em primeiro lugar, assume-se que $\alpha, \beta, \gamma$ não se modificam com os diferentes regimes. Essa premissa, presente em todos os trabalhos de IH, será testada para verificar se é, de fato, aceitável. Além disso, considerar-se-á que $\sigma_{z}^{2}$ é homocedástico. Essa simplificação não é muito restritiva tendo em vista que o modelo incluirá dois fatores observáveis que não apresentam qualquer restrição.

Aplicando as premissas anteriores, é possível obter uma identificação parcial com três regimes, recuperando os valores de $\alpha$ e $\beta$ a partir das equações quadráticas mostradas a seguir. 


$$
\begin{aligned}
a & =\left(\Omega_{1,2}-\Omega_{1,1}\right)\left(\Omega_{12,3}-\Omega_{12,1}\right) \\
& -\left(\Omega_{12,2}-\Omega_{12,1}\right)\left(\Omega_{1,3}-\Omega_{1,1}\right) \\
a \alpha^{2}+b \alpha+c=0, \text { onde } b & =\left(\Omega_{2,2}-\Omega_{2,1}\right)\left(\Omega_{1,3}-\Omega_{1,1}\right) \\
& -\left(\Omega_{1,2}-\Omega_{1,1}\right)\left(\Omega_{2,3}-\Omega_{2,1}\right) \\
c & =\left(\Omega_{12,2}-\Omega_{12,1}\right)\left(\Omega_{2,3}-\Omega_{2,1}\right) \\
& -\left(\Omega_{2,2}-\Omega_{2,1}\right)\left(\Omega_{12,3}-\Omega_{12,1}\right) \\
a \beta^{2}+b . \beta+c=0, \text { onde } a & =\left(\Omega_{2,3}-\Omega_{2,1}\right)\left(\Omega_{12,2}-\Omega_{12,1}\right) \\
& -\left(\Omega_{2,2}-\Omega_{2,1}\right)\left(\Omega_{12,3}-\Omega_{12,1}\right) \\
b & =\left(\Omega_{2,2}-\Omega_{2,1}\right)\left(\Omega_{1,3}-\Omega_{1,1}\right) \\
& -\left(\Omega_{1,2}-\Omega_{1,1}\right)\left(\Omega_{2,3}-\Omega_{2,1}\right) \\
c & =\left(\Omega_{12,3}-\Omega_{12,1}\right)\left(\Omega_{1,2}-\Omega_{1,1}\right) \\
& -\left(\Omega_{12,2}-\Omega_{12,1}\right)\left(\Omega_{1,3}-\Omega_{1,1}\right)
\end{aligned}
$$

A matriz de covariância dos resíduos é definida como

$$
\Omega=\left(\begin{array}{cc}
\Omega_{1, j} & \Omega_{12, j} \\
& \Omega_{2, j}
\end{array}\right)
$$

onde j é o índice que representa os regimes.

As equações têm solução real se a matriz A for inversível. Nesse caso, existem dois valores possíveis para $\alpha$ e $\beta$ sendo que uma das raízes é a solução real e a outra, sua inversa. A solução real deve ser escolhida em função da magnitude e do sinal teóricos esperados. 